\title{
DIFFUSIVE PROPAGATION OF PHONON BEAMS IN YTTRIUM ALUMINUM GARNETS CONTAINING SUBSTITUTIONAL RARE EARTH ATOMS
}

\author{
S.N. IVANov ${ }^{a}$, E.N. KhazANOV ${ }^{a}$, T. PASZKIEWICZ ${ }^{b}$, A.V. TARANOV ${ }^{a}$ \\ AND M. WILCZYŃSKI ${ }^{b}$ \\ ${ }^{a}$ Institute of Radioengineering and Electronics, Russian Academy of Sciences \\ Mokhovaya 11, Moscow, GSP-3, 103907, Russia \\ ${ }^{b}$ Institute of Theoretical Physics, University of Wrocław \\ Pl. M. Borna 9, 50-204 Wrocław, Poland
}

The diffusive maxima of phonon signals are studied for a number of solid solutions of rare earth atoms in yttrium aluminum garnets. The used exact formula for the diffusion constants allows for qualitative discussion of the obtained results. The established energy of phonons, forming the diffusive maximum of phonon signal of the temperature $T_{\mathrm{H}}$ arriving at the bolometer, ranges from $3.2 k_{\mathrm{B}} T_{\mathrm{H}}$ to $4.2 k_{\mathrm{B}} T_{\mathrm{H}}$, which is in reasonable agreement with the existing estimations. The qualitative analysis allows us to estimate the contribution, made by the rare earth ions occupying the octahedral positions of the sixfold oxygen coordination, to the scattering of phonons due to lattice imperfections in yttrium aluminum garnets.

PACS numbers: $63.20 . \mathrm{Mt}, 66.70 .+\mathrm{f}, 05.60 .+\mathrm{w}$

\section{Introduction}

There is a number of experimental and theoretical studies which extend our knowledge of the peculiarities of the nonequilibrium phonon propagation in imperfect crystalline media, in particular in crystals which contain point mass defects. The most detailed theoretical treatment of this problem, taking also into account the phonon-phonon interactions, has been given by Levinson and collaborators (cf. [1]). The experimental results can be found in [2-4].

However, some unanswered questions still remain. One of them is related to the spectral composition of phonons radiated by a thin metallic film into the studied crystal. Besides, the existing theoretical description of the phonon propagation in imperfect crystals is merely semiquantitative and based on simple estimations. Therefore a careful comparison of the experimental results with the theoretical calculations is needed. 
The purpose of the present paper is the comparison of the implications of the previously derived expression for the diffusion constant [5] with the experimental findings. In particular, here we study the dependence of the measured diffusion constant on the heater temperature for a number of yttrium-aluminum garnets containing the rare earth substitution atoms of $R$ type $(R=D y, G d, L u, Y b)$.

The YAG:R crystals are well suited for such studies since due to the closeness of the ionic radii of yttrium and rare earths, the presence of substitutional rare-earth atoms does not distort the crystalline lattice and consequently the elastic constants remain unaffected by their presence. At such conditions the prevailing phonon scattering mechanism is the elastic scattering by point mass defects. Masses of $\mathrm{Y}$ and $\mathrm{R}$ atoms differ substantially, which results in large values of the scattering coefficient $g\left(g \approx 10^{-4} \div 10^{-3}\right)$. Therefore, one can observe the diffusive motion of low energy nonequilibrium phonons almost undisturbed by inelastic processes.

As it is seen from the chemical formula for such solid solutions $\left(\mathrm{Y}_{3-x} \mathrm{R}_{x} \mathrm{Al}_{2-y} \mathrm{R}_{y}\left(\mathrm{AlO}_{4}\right)_{3}\right)$, the rare-earth ions can occupy two nonequivalent positions in the garnet lattice, which contain eight of these formula units per unit cell. The occupancy of the tetrahedral sites of Al by rare-earth ions (A-centers) can be quite large, especially in the case of Lu and $\mathrm{Yb}$ ions. As their masses are much larger than the mass of $\mathrm{Al}$ ions, the related scattering of phonons can be quite strong. Here we shall give a quantitative estimation of the effectiveness of the scattering of low energy acoustic phonons by these A-centers.

\section{Chaotization of the initial distribution of phonons}

Let us assume that we deal with a perfect massive crystalline specimen which contains some amount of substitutional atoms of almost the same ionic radii as the host atoms, but with the atomic masses substantially different from the host ones. Further on, we assume that the ambient temperature $T$ is much lower than the Debye temperature $\Theta_{D}$ of the crystal, therefore the density of thermal phonons is very small.

The specimen has the form of a slab, and we assume that the thickness $L_{z}$ of it is much smaller than its perpendicular dimensions $L_{x}$ and $L_{y}$. Nonequilibrium phonons are excited by heating a thin metallic film on the end face of the sample. Assuming that the film attains the local thermal equilibrium with the temperature $T_{\mathrm{H}}$ slightly higher than the ambient temperature $T$, the distribution of the injected phonons is expected to be Planckian. For small power density released in the heater mostly the long wavelength acoustic phonons (LAPs) are generated. Assume that the spectral distribution of the injected phonons attains the maximum for the frequency $\omega=\omega_{\mathrm{H}} \equiv \omega_{\mathrm{m}}\left(T_{\mathrm{H}}\right)$, where

$$
\omega_{\mathrm{m}}\left(T_{\mathrm{H}}\right)=\frac{\xi k_{\mathrm{B}} T_{\mathrm{H}}}{\hbar} \text {. }
$$

For the Planck distribution function $\xi=\xi_{\mathrm{Pl}} \equiv 2.82$.

An initially ballistic pulse of the injected phonons is gradually chaotized due to scattering of phonons by substitutional atoms. Consequently, the ballistic 
component of the pulse steadily diminishes, while the diffusive one grows. This process can be studied in details for isotropic media (cf. $[6,7]$ ).

\section{Long-time asymptotics of the distribution function}

We restrict ourselves to the description of the long-time asymptotics of the chaotization process of an initial distribution. At this stage of evolution the state of nonequilibrium phonon gas is described by the phonon number density $n(r, t)$.

For long wavelength acoustic phonons the frequency of a phonon $K=(k, j)$ is a linear function of the length $k \equiv|k|$ of the wave vector $k$ :

$$
\omega(K)=c(\hat{K}) k,
$$

where $\hat{K}$ stands for the pair $(\hat{k}, j), \hat{k}$ being the direction of the phonon wave vector $(\hat{k}=k /|k|)$ and $j$ enumerates polarizations $(j=0,1,2)$.

The Debye velocity $c_{D}$ is defined by the following condition:

$$
\langle 1\rangle_{\hat{K}}=1 \text {, }
$$

where $\langle\ldots\rangle_{\hat{K}}$ denotes the mean value, e.g. for an arbitrary function $A(\hat{K})$ :

$$
\langle A(\hat{K})\rangle_{\hat{K}}=\frac{1}{3} \sum_{j=0}^{2} \int_{4 \pi} \frac{\mathrm{d} \hat{k}}{4 \pi}\left[\frac{c_{\mathrm{D}}}{c(\hat{K})}\right]^{3} A(\hat{K}) .
$$

The Debye temperature $\left(T \ll \Theta_{\mathrm{D}}\right)$ is related to the Debye velocity

$$
\Theta_{\mathrm{D}}=\hbar c_{\mathrm{D}} a^{-1} / k_{\mathrm{B}}
$$

$a$ being the lattice constant.

For spatially inhomogeneous states of a medium phonons should be understood as the wave packets, which move with the group velocity $v$. For LAPs the velocity $\boldsymbol{v}$ depends only on $\hat{K}$.

The number density $n(r, t)$ of phonons propagating in a cubic medium obeys the diffusion equation [5]

$$
\partial n(\hat{K}, r, t) / \partial t \approx D \partial^{2} n(\hat{K}, r, t) / \partial r^{2},
$$

where $D$ is the diffusion constant

$$
D=\frac{1}{3} \tau(\omega)\left\langle v^{2}(\hat{K})\right\rangle_{\hat{K}}
$$

The parameter $\tau(\omega)$ is the Rayleigh time characterizing the scattering of LAPs by substitutional atoms, which depends on the phonon frequency, on the abundance and on the differences of the host and the substitutional atoms masses.

The chemical structural formula for the yttrium aluminum garnet can be written as $\mathrm{Y}_{2} \mathrm{Al}_{2} \mathrm{Al}_{3} \mathrm{O}_{12}$ (i.e. $\left.s=20\right)$ with eight $(n=8)$ of these formula units per unit cell. The yttrium atoms occupy 24 dodecahedral [C]-sites, the aluminum atoms occupy 16 octahedral ([A]-sites) and 24 dodecahedral sites. The remaining 96 sites are occupied by the oxygen atoms. The substitutional atoms can occupy dodecahedral [C]- as well as octahedral [A]-sites. Let us denote the abundance of the substitutional atoms in the [A]-sites by $x\left(x \equiv f_{\text {oct }}\right)$ and in the [C]-sites by $y$ 
$\left(y \equiv f_{\text {dod }}\right)$. Then one can write the factor $g$ for the yttrium aluminum garnet in the following form:

$$
g=\frac{8 x\left(1-\frac{x}{3}\right)\left(M_{\mathrm{R}}-M_{\mathrm{Y}}\right)^{2}+8 y\left(1-\frac{y}{2}\right)\left(M_{\mathrm{R}}-M_{\mathrm{Al}}\right)^{2}}{\left(8 \overline{M_{\mathrm{CFU}}}\right)^{2}},
$$

where

$$
\overline{M_{\mathrm{CFU}}}=\frac{1}{20} \sum_{\alpha=1}^{20} \overline{M_{\alpha}} .
$$

The Rayleigh characteristic time $\tau$ is proportional to the factor $g$ (cf. $[6,5]$ ):

$$
\tau^{-1} \equiv \tau^{-1}(\omega)=\frac{v_{0} g \omega^{4}}{4 \pi c_{\mathrm{D}}^{3}}
$$

where $v_{0}$ is the volume of the unit cell.

According to the experimental conditions (cf. Sec. 2) the sample under consideration has a form of a thin slab. Let us fix our coordinate system so that the $z$-axis is perpendicular to the slab. Two remaining axes are lying on the surface of the slab and are arbitrarily oriented. Because of the chosen specimen geometry the phonon number density only weakly depends on the $x$ and $y$ coordinates and the diffusion equation simplifies.

At the ambient temperature $T$ much lower than the Debye temperature $\Theta_{D}$ one can neglect the presence of thermal phonons. As we previously noted (cf. Eq. (1)), the frequencies of the dominating part of the injected phonons depend on the heater temperature $T_{\mathrm{H}}$. Assuming that the source produces short pulses of phonons of the frequency $\omega_{\mathbf{H}}$ at one of the slab surfaces, one obtains the familiar expression

$$
n\left(\omega_{\mathrm{H}}, z, t\right) \sim \frac{1}{\sqrt{D \pi t}} \exp \left[-z^{2} / 4 D\left(\omega_{\mathrm{H}}\right) t\right] .
$$

Assume that we generate a pulse of phonons at the instant $t=0$. After a lap of time $t$ much larger than $\tau\left(\omega_{\mathrm{H}}\right)$, at the opposite face of the slab, one observes a signal which attains its maximum at

$$
t_{\mathrm{m}}=\frac{L_{z}^{2}}{4 D\left(\omega_{\mathrm{H}}\right) A}
$$

where $A=1 / 2$. For a point source the coefficient $A$ assumes the value of $3 / 2$. Generally, the solution of the diffusion equation (5) has the form of a convolution of the initial function $n(x, y, t=0) \equiv h(x, y)$ with the function (10) depending now on $r^{2}$. However, since in the experiment the signal is averaged over the slab surface, when the dependency of $h(x, y)$ on the spatial variables is weak, one can expect that the formula (11) is still valid with $1 / 2 \leq A \leq 3 / 2$.

\section{Discussion}

The dependence of the diffusion coefficient $D$ on the heater temperature $T_{\mathrm{H}}$ is shown for a number of different specimens in Fig. 1. The upper line, labeled as 1 corresponds to the results of measurements of the arrival time $t_{\mathrm{m}}$ for two 


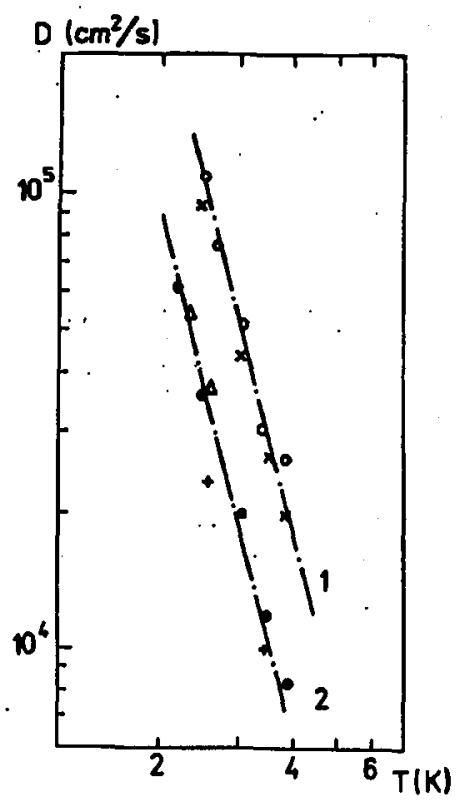

Fig. 1. The diffusion coefficient vs. the heater temperature $T_{\mathrm{H}}$ (dots) for 1 $\mathrm{Lu}_{3} \mathrm{Al}_{5} \mathrm{O}_{12}, 2-\mathrm{Y}_{1} \mathrm{Lu}_{2} \mathrm{Al}_{5} \mathrm{O}_{12}$. The solid lines represent the results of calculation.

pure $\mathrm{Lu}_{3} \mathrm{Al}_{5} \mathrm{O}_{12}$ specimens. The diffusion coefficient was calculated with the use of Eqs. (7), (8), (9) and (11). We assumed that phonons are scattered by the lutetium atoms substituting the aluminum atoms in the tetrahedral sites.

The curve no. 1 is consistent with the theoretically predicted $D \sim T_{\mathbf{H}}^{-4}$ dependence. We also estimated the value of the coefficient $\xi \approx 3.2$ (cf. Eq. (1)).

The lower curve in Fig. 1 corresponds to the experimentally measured arrival times for three samples of $\mathrm{Y}_{1} \mathrm{Lu}_{2} \mathrm{Al}_{5} \mathrm{O}_{12}$ solid solutions (i.e. the matrix is a pure lutetium aluminum garnet). For this matrix there are two scattering mechanisms. The residual one is related to some amount of foreign substitutional impurities as well as of oxygen vacancies. An additional scattering mechanism is related to the substitutional $\mathrm{Lu}$ atoms placed in the tetrahedral aluminum sites. It was previously established that the concentration of the Lu atoms in the octahedral A-sites in pure $\mathrm{Lu}_{3} \mathrm{Al}_{5} \mathrm{O}_{12}$ matrices and in $\mathrm{Y}_{1} \mathrm{Lu}_{2} \mathrm{Al}_{5} \mathrm{O}_{12}$ lattices are almost the same. Therefore, the comparison of phonon scattering in $\mathrm{Y}_{1} \mathrm{Lu}_{2} \mathrm{Al}_{5} \mathrm{O}_{12}$ and $\mathrm{Lu}_{3} \mathrm{Al}_{5} \mathrm{O}_{12}$ crystals allows us to establish the contribution to scattering made by the lutetium atoms placed in the dodecahedral [C]-sites. As previously, the measurements confirmed the linear dependence of $D$ on the $T_{\mathbf{H}}^{-4}$ and gave the value of the $\xi$ coefficient equal to 3.29 .

Similar considerations with the subtraction of the residual scattering term were done for results of measurements on other specimens (e.g. for $\mathrm{Y}_{2.65} \mathrm{Lu}_{0.35} \mathrm{Al}_{5} \mathrm{O}_{12}, \mathrm{Y}_{2.7} \mathrm{Dy}_{0.3} \mathrm{Al}_{5} \mathrm{O}_{12}, \mathrm{Y}_{2.65} \mathrm{Gd}_{0.15} \mathrm{Yb}_{0.5} \mathrm{Al}_{5} \mathrm{O}_{12}$ ). We accounted for substitutional rare earth atoms in tetrahedral A-positions. The results confirmed the Rayleigh dependence of the diffusion coefficient on $T_{\mathrm{H}}$ with $\xi$ not exceeding 4.1 . 
Krasilnikov and Kozorezov [7] studied a model of an isotropic medium, containing Rayleigh scattering centers. They derived a formula for the time dependence of the energy density of phonons at an arbitrarily chosen point. From this formula it follows that the signal maximum is formed mainly by phonons of the energy $\hbar \omega_{\mathrm{m}}\left(T_{\mathrm{H}}\right) \sim 4 k_{\mathrm{B}} T_{\mathrm{H}}$.

We conclude that the experiments previously performed and the theoretical model calculations indicate that the maximum of the diffusive signal is formed by phonons of the energy bigger than the energy corresponding to the maximum of the Planck distribution function at a given heater temperature. Our experimental results entirely confirm this fact and the obtained values of the coefficient $\xi$ reasonably agree with the results obtained by the above-mentioned authors.

We thank the State Committee for Scientific Research (Republic of Poland) for support for this work through a contract No. 397/2/91 and for support of our visit in Wroclaw of two of us (S.N.I. and A.V.T.).

\section{References}

[1] Y.B. Levinson, in: Nonequilibrium Phonons in Nonmetallic Crystals, Eds. W. Eisenmenger, A.A. Kaplyanskii, North-Holland, Amsterdam 1986, p. 91.

[2] S.N. Ivanov, E.N. Khazanov, A.V. Taranov, Sov. Phys.-JETP 62, 1051 (1985).

[3] S.N. Ivanov, E.N. Khazanov, A.V. Taranov, Sov. Phys.-Solid State 29, 385 (1987).

[4] S.N. Ivanov, E.N. Khazanov, A.V. Taranov, Sov. Phys.-JETP 72, 731 (1991).

[5] T. Paszkiewicz, M. Wilczyński, Z. Phys. B, Condens. Matter 88, 5 (1992).

[6] Jasiukiewicz, T. Paszkiewicz, Z. Phys. B, Condens. Malter 77, 209 (1989).

[7] A.G. Kozorezov, M.V. Krasilnikov, Fiz. Tverd. Tela 31, 109 (1989). 